\section{Silber gegen anale Strahlendermatitis}

\begin{abstract}
Dermatitis entsteht fast immer, wenn Patienten mit Anal- oder Rektumkarzinom eine Chemoradiotherapie erhalten. Eine Prophylaxe mit einem ursprünglich für Brandwunden entwickelten Silbergewebe soll sie lindern.
\end{abstract}

- $\ddot{\mathrm{u}}$ ür Patienten mit einem Karzinom des Analkanals oder mit fortgeschrittenem Rektumkarzinom ist die Chemoradiotherapie eine kurative Möglichkeit oder zumindest eine wichtige Ergänzung zur Operation. Doch fast alle Patienten entwickeln eine strahleninduzierte Dermatitis (SID) - in etwa der Hälfte bis drei Viertel der Fälle von hohem Schweregrad. Neben der effektiven Behandlung ist daher eine Prävention von großer Bedeutung für die Lebensqualität der Patienten.

In einer Phase-III-Studie wurde der Nutzen des „Silver Clear Nylon Dressing " (SCND) getestet. Der nicht adhärente, nanokristalline Verbandstoff wur- de zunächst zur Abdeckung von Brandwunden entwickelt. Sein Kern besteht aus absorbierendem Rayon und Polyester und wird auf beiden Seiten von einer aufgedampften Silberschicht bedeckt. Deren antimikrobielle Aktivität verbessert die Wundheilung.

42 Patienten mit einem Karzinom von Rektum oder Analkanal wurden mit 50,4-59,5 Gy bestrahlt und randomisiert: Die Behandlung mit SCND dauerte von Tag 1 bis zwei Wochen nach Ende der Radiotherapie. Im Kontrollarm wurde Sulfadiazinsalbe gegeben, sobald eine Dermatitis auftrat. Digitale Fotos, zwei Wochen vor, am letzten Tag sowie zwei Wochen nach der Radiotherapie aufge- nommen, wurden verblindet von zehn Ärzten unter Verwendung der CTC-Kriterien für eine Dermatitis bewertet.

Am letzten Tag der Bestrahlung, wenn das Ausmaß der SID am größten ist, lag der mittlere Dermatitis-Score bei 2,53 in der Kontrollgruppe und bei 1,67 für den SCND-Arm. Zwei Wochen später betrug der Unterschied noch 0,39 Punkte zugunsten der SCND-Therapie $(p=0,39)$.

Fazit: Die Behandlung mit dem SCND reduziert die SID bei Patienten mit Karzinomen von Rektum und Analkanal, die eine kombinierte Chemo- und $\mathrm{Ra}$ diotherapie erhalten. Das Material lässt sich in Unterwäsche einarbeiten, die ohne toxische Nebenwirkungen getragen werden kann.

Christina Berndt

Niazi TM et al. Silver clear nylon dressing is effective in preventing radiation-induced dermatitis in patients with lower gastrointestinal cancer: Results from a phase III study. Int J Radiat Oncol Biol Phys. 2012;84(3):e305-10.

\title{
CRC: Magnesium-Verlust verkürzt das Leben
}

\section{Der oft drastische Abfall des Serum- Magnesiums unter Cetuximab schien sich in einigen Studien güns- tig auf das Überleben auszuwirken. Ob sich das in einer prospektiven Untersuchung bewahrheitet?}

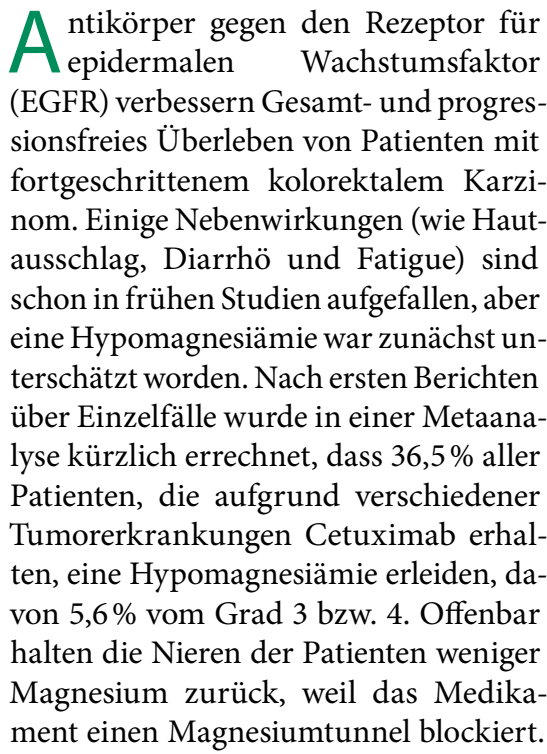

Verschiedene Studien gaben dann Hinweise darauf, dass Patienten mit starkem Abfall an Serum-Magnesiumspiegeln länger (auch krankheitsfrei) leben. In einer aktuellen prospektiven Studie wurde dieser Frage nun noch einmal nachgegangen.

511 Patienten mit vorbehandeltem, fortgeschrittenem kolorektalem Karzinom erhielten „Best Supportive Care“ mit oder ohne Cetuximab. Vor Therapiebeginn und an Tag 28 wurden die Magnesiumspiegel bestimmt und mit dem Outcome abgeglichen. Dabei wurden die Patienten mit Hypomagnesiämie vom Grad $\geq 1$ jenen ohne Hypomagnesiämie $(\mathrm{Grad}=0)$ gegenübergestellt. Auch wurden Gruppen mit einer Magnesiumreduktion von $<20 \%$ bzw. $\geq 20 \%$ gebildet.

Die mittlere prozentuale Reduktion von Magnesium an Tag 28 betrug $10 \%$ $(-42,4-63,0 \%)$ für die 260 Patienten der Cetuximabgruppe gegenüber $0 \%(-21,1-$ $25 \%)$ für die 251 Patienten der Kontrollgruppe $(\mathrm{p}<0,0001)$. Ein Schweregrad an Hypomagnesiämie von $\geq 1$ oder eine mehr als $20 \%$ ige Reduktion waren assoziiert mit einem schlechteren Gesamt- überleben. Die Risikoquotienten (HR) betrugen 1,61 bzw. 2,08. In multivariaten Analysen, die auch den Hautausschlag einbezogen, war Dyspnoe (vom Grad $\geq 3$ ) bei Patienten mit einer Magnesiumreduktion $\geq 20 \%$ häufiger als bei solchen mit geringerer Reduktion: 68 vs. $45 \%$ der Patienten waren betroffen. Darüber hinaus trat auch Anorexie vom Grad 3 bzw. 4 häufiger bei Patienten mit Hypomagnesiämie vom Grad $\geq 1$ auf: 81 vs. $63 \%$.

Fazit: Im Gegensatz zu früheren Berichten war in dieser prospektiven Studie die durch Cetuximab hervorgerufene Hypomagnesiämie keineswegs förderlich, sondern dem Gesamtüberleben der Patienten abträglich. Das galt auch, wenn für den Grad des Hautausschlags adjustiert wurde. Das Ausmaß der Hypomagnesiämie ist also schwerwiegender und hat einen größeren prognostischen Wert als das Ausmaß des Hautausschlags.

Christina Berndt

Vickers MM et al. Association of hypomagnesemia with inferior survival in a phase III, randomized study of cetuximab plus best supportive care versus best supportive care alone: NCIC CTG/AGITG CO.17. Ann Oncol. 2013;24(4):953-60. 